\title{
Recurrent UTIs and cystitis symptoms in women
}

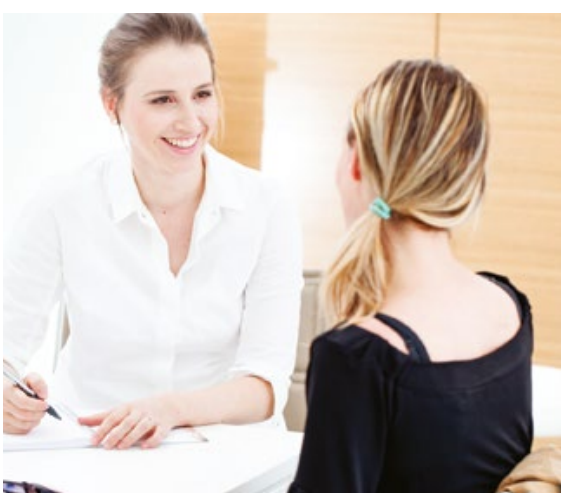

CPD

Karen McKertich, Uri Hanegbi

\section{Background}

Recurrent urinary tract infections (rUTIs) and recurrent cystitis symptoms without infection occur commonly in women and present frequently in general practice.

\section{Objective}

The aim of this article is to provide a management approach to the assessment and treatment of recurrent cystitis symptoms in women with rUTIs as well as women who have negative urine cultures.

\section{Discussion}

Five common clinical scenarios are discussed with different approaches to treatment: true rUTIs with positive urine cultures, women with variable urine cultures (some positive and some negative), women with negative urine cultures who have pyuria +/- haematuria, women with completely normal urine cultures and women with ongoing symptoms after a definite UTI. Red flags signalling the need for early referral to a urologist for further assessment are discussed. Both non-antibiotic and antibiotic-related strategies to treat women with rUTIs are available.
URINARY TRACT INFECTIONS (UTIS) are an extremely common problem in women and are a cause of great personal morbidity as well as cost to the health system. While an occasional UTI is simple to treat, recurrent UTIs (rUTIs) and cystitis with variable or negative urine cultures are a complex diagnostic and therapeutic challenge. In this article, the term 'cystitis' is defined as irritative voiding symptoms that are related to infection, non-infective inflammation and symptoms in the absence of bladder inflammation.

\section{Classification of UTIs in women} An rUTI is defined as $\geq 2$ episodes of UTI within six months or $\geq 3$ or episodes of UTI within 12 months with the isolation of $>10^{3} \mathrm{CFU} / \mathrm{mL}^{1}$

\section{Non-complicated versus complicated UTIs}

UTIs can also be categorised as complicated or non-complicated. The aim of investigations is to define and correct complicating factors that predispose to recurrence of UTIs.

In general, an episode of acute non-complicated UTI occurs in an otherwise healthy, non-pregnant woman who is not known to have an abnormality in her urinary tract (Table 1). The most common cause of acute uncomplicated cystitis is Escherichia coli in $70-95 \%$ of cases. $^{2}$
Complicated UTIs occur in women who have underlying abnormalities of their urinary tract anatomy or physiology (Table 1). Women with complicated rUTIs require referral for further assessment.

\section{Definitions of UTI}

Controversies exist regarding the bacteriological definition of UTI. In 1960, Kass defined significant bacteriuria as $>10^{5} \mathrm{CFU} / \mathrm{mL},{ }^{3}$ but it is now recognised that in symptomatic women, $E$. coli counts of $>10^{2} \mathrm{CFU} / \mathrm{mL}$ can accurately confirm bladder bacteriuria. ${ }^{4}$ The dilutional effect of a high fluid intake at the time of UTI on the accuracy of culture results and concentration threshold for UTI is not well understood. E. coli in mixed flora in midstream urine (MSU) is also predictive of bladder bacteriuria in symptomatic women and should not be considered a contaminant. ${ }^{4}$ This finding merits treatment in symptomatic women. Asymptomatic bacteriuria is the term used when the standard urine culture detects a uropathogen $>10^{5} \mathrm{CFU} / \mathrm{mL}$ in an individual with no lower urinary tract symptoms.

The importance of the MSU in rUTIs cannot be underestimated. The MSU defines whether the patient has a bacterial UTI as opposed to other potential causes of cystitis (Table 2) and is also fundamental to ensure 
optimal and accurate treatment with the appropriate antibiotic.

Five common clinical scenarios of 'cystitis': Is it a recurrent UTI? Cystitis refers to irritative voiding symptoms (dysuria or pain after micturition), frequency, urgency, cloudy or offensive urine and discomfort or pain in the bladder, urethra or vagina.

There are five clinical scenarios that general practitioners (GPs) face when treating women with cystitis, each requiring a specific approach to diagnosis and management (Figure 1):

1. Women with confirmed rUTIs (positive urine cultures)

2. Women with symptoms of cystitis with variable urine cultures (both positive and negative urine cultures)

3. Women with symptoms of cystitis and negative urine cultures with microhaematuria +/- micropyuria

4. Women with symptoms of cystitis with normal urine cultures (no pyuria or haematuria)

5. Women with ongoing symptoms after a definite UTI (positive urine culture) who now have a negative urine culture.

\section{Women with confirmed recurrent UTIs (positive urine cultures)}

\section{A. Assessment of women with confirmed recurrent UTIs}

\section{History}

The acute onset of dysuria and frequency in a young woman in the absence of concomitant vaginal irritation or vaginal discharge gives a $90 \%$ probability of UTI. ${ }^{5}$

History-taking should elucidate red flags for rUTIs (Box 1) as well as factors causing complicated UTIs.

\section{Physical examination}

Abdominal and pelvic examination should be performed to look for anatomical or functional abnormalities of the urinary tract including atrophic vaginal change and pelvic organ prolapse. ${ }^{6}$

\section{Urine cultures}

Performance of urine cultures when women are symptomatic is crucial in the assessment and management of rUTIs. ${ }^{6}$ The results of urine cultures confirm the diagnosis, provide antibiotic sensitivities, allow targeted treatment of the UTI, confirm whether the treatment approach is effective and guide choice of antibiotics for prophylaxis. A lack of correlation between MSUs and the clinical diagnosis also raises a red flag to consider alternative diagnoses (Box 1).

It is recommended that each UTI episode is clinically evaluated as a separate event requiring culture, with consideration given to a catheterised specimen if a question of contamination of the urine specimen is raised by a high epithelial cell count. ${ }^{6}$

\section{Urinary tract imaging}

While investigations in an otherwise healthy women with uncomplicated rUTIs have a low diagnostic yield, ${ }^{6}$ a simple non-invasive test such as renal ultrasonography will identify the small proportion of women who have an anatomical abnormality.

Women who fall outside the category of uncomplicated rUTIs (Table 1) and who have a suspicion of complicating factors should have renal tract imaging commencing with renal tract ultrasonography, which provides information about both the kidneys and bladder emptying.

\section{Cystoscopy}

Cystoscopy can be performed under local anaesthesia as an ambulatory procedure in the urologist's rooms or under general anaesthesia.

Cystoscopy is of low yield in the index patient (an otherwise healthy adult non-pregnant premenopausal woman $)^{7}$ with uncomplicated rUTIs but should be

\begin{tabular}{|c|c|c|}
\hline \multirow[t]{5}{*}{$\begin{array}{l}\text { Structural abnormalities } \\
\text { of the urinary tract }\end{array}$} & Congenital & $\begin{array}{l}\text { - Vesicoureteral reflux } \\
\text { - Pelviureteric junction obstruction } \\
\text { - Polycystic kidney disease }\end{array}$ \\
\hline & Obstruction & $\begin{array}{l}\text { - Calculi - renal, ureteric or bladder } \\
\text { - Bladder outlet obstruction } \\
\text { - Ureteric/urethral stricture }\end{array}$ \\
\hline & Neoplastic & - Bladder tumour \\
\hline & Functional & $\begin{array}{l}\text { - Urinary tract instrumentation } \\
\text { - Foreign body eg urethral catheter, } \\
\text { - Ineteric stent } \\
\text { - Untermittent self-catheterisation } \\
\text { - Urinary diversion } \\
\text { - Urinary or faecal incontinence } \\
\text { - Poor bladder emptying/increased } \\
\text { post-void residual }\end{array}$ \\
\hline & Neurological & $\begin{array}{l}\text { - Neurological disease affecting the } \\
\text { urinary tract eg: } \\
\text { - multiple sclerosis } \\
\text { - Parkinson's disease } \\
\text { - spinal cord injury } \\
\text { - peripheral neuropathy } \\
\text { - diabetes }\end{array}$ \\
\hline General conditions & & $\begin{array}{l}\text { - Pregnancy } \\
\text { - Diabetes } \\
\text { - Immunosuppression } \\
\text { - Renal failure } \\
\text { - Renal transplant }\end{array}$ \\
\hline Demographic factors & & - Hospital-acquired infection \\
\hline
\end{tabular}


considered even in the index patient when atypical features are present or the patient fails to respond to treatment.

Cystoscopy should be considered early in the assessment process for all other patients with complicating factors or with red flags for early referral to a urologist (Box 1).

\section{Urodynamic studies}

Other tests such as a urodynamic study are reserved for women in whom other diagnoses are considered, including neurogenic bladder, and in those at high risk of bladder complications from previous treatments (eg bladder outlet obstruction from previous incontinence surgery and pelvic radiotherapy).

\section{B. General advice for prevention of recurrent UTIs}

It is recommended that the patient and doctor engage in a shared decisionmaking process that includes a discussion of the risks and benefits of all management options before embarking on a management plan tailored to the individual woman. ${ }^{6}$

\section{Increased fluid intake}

While the advice to increase fluid intake to prevent infection is frequently given, there is little evidence to support it. ${ }^{8}$ It is, however, a low-cost and low-risk intervention.

\section{Sexual practices}

Postcoital voiding - The relationship between intercourse and UTI is controversial, with variable study findings. ${ }^{9,10}$ A simple measure such as postcoital voiding can be recommended in women who note intercourse as a trigger for their UTIs.

Contraceptive use-Spermicide or diaphragm contraceptive device usage should be avoided as these have been shown to increase UTI risk. ${ }^{11}$

\section{Risk factors that cannot be changed} Genetic predisposition - There is evidence for a genetic predisposition to rUTIs in some women with risk factors including age at first infection of $<15$ years and having a mother who has a history of UTIs. ${ }^{12}$
Table 2. Differential diagnosis of cystitis symptoms in women with negative urine cultures

\begin{tabular}{|c|c|}
\hline $\begin{array}{l}\text { Urinary tract infection } \\
\text { with false-negative } \\
\text { midstream urine } \\
\text { (MSU) test }\end{array}$ & $\begin{array}{l}\text { - Low bacterial count below the threshold for laboratory } \\
\text { reporting } \\
\text { - Low bacterial count due to dilution from high fluid intake } \\
\text { - Specimen interpreted as contaminated } \\
\text { - Difficult-to-culture urinary pathogen } \\
\text { - Antibiotics taken prior to MSU }\end{array}$ \\
\hline Infectious & $\begin{array}{l}\text { - Urethritis } \\
\text { - Sexually transmitted infection eg Chlamydia trachomatis, } \\
\text { Neisseria gonorrohoea, herpes simplex } \\
\text { - Vulvovaginitis } \\
\text { - Cervicitis }\end{array}$ \\
\hline Dermatological & $\begin{array}{l}\text { - Atrophic vaginitis } \\
\text { - Dermatitis - contact or irritant } \\
\text { - Lichen sclerosus } \\
\text { - Psoriasis } \\
\text { - Other vaginal skin conditions }\end{array}$ \\
\hline Non-infectious & $\begin{array}{l}\text { - Excessive fluid intake } \\
\text { - Stone eg distal ureteric calculus or bladder calculus } \\
\text { - Overactive bladder syndrome } \\
\text { - Interstitial cystitis/bladder pain syndrome } \\
\text { - Pelvic floor muscle dysfunction } \\
\text { - Endometriosis } \\
\text { - Urethritis eg reactive arthritis/Behçet's disease }\end{array}$ \\
\hline Neoplastic & $\begin{array}{l}\text { - Bladder cancer - transitional cell carcinoma or carcinoma in } \\
\text { situ of bladder } \\
\text { - Renal cancer causing haematuria } \\
\text { - Urethral cancer } \\
\text { - Metastatic cancer } \\
\text { - Adjacent cancer infiltrating bladder } \\
\text { - Vaginal or vulvar cancer }\end{array}$ \\
\hline $\begin{array}{l}\text { Structural - within } \\
\text { the urinary tract }\end{array}$ & $\begin{array}{l}\text { - Urethral diverticulum } \\
\text { - Periurethral/Skene's duct cyst } \\
\text { - Urethral irritation after intercourse } \\
\text { - Urethral stricture } \\
\text { - Pelvic organ prolapse - high-grade cystocele or other prolapse }\end{array}$ \\
\hline $\begin{array}{l}\text { Structural - adjacent } \\
\text { to the urinary tract }\end{array}$ & $\begin{array}{l}\text { - Gastrointestinal tract pathology - diverticulitis, diverticular } \\
\text { abscess, vesicoenteric fistula } \\
\text { - Ovarian tumour } \\
\text { - Other pelvic mass }\end{array}$ \\
\hline Trauma/latrogenic & $\begin{array}{l}\text { - Ureteric stent } \\
\text { - Foreign body in the urinary tract related to previous pelvic } \\
\text { surgery eg mesh sling procedure, mesh prolapse repair, suture } \\
\text { from Burch colposuspension } \\
\text { - Genitourinary surgery or instrumentation eg urinary catheter } \\
\text { - Pelvic radiation } \\
\text { - Perineal trauma eg post-intercourse, associated with bike } \\
\text { riding or horseback riding }\end{array}$ \\
\hline Medication related & $\begin{array}{l}\text { - Spermicides } \\
\text { - Topical deodorants or detergents } \\
\text { - Cyclophosphamide cystitis } \\
\text { - Ketamine cystitis }\end{array}$ \\
\hline
\end{tabular}


Anatomical factors - A shorter distance between the urethral meatus and anus has been shown in some young women with rUTIs. ${ }^{13}$

\section{Antibiotic treatment options for women with confirmed recurrent UTIs}

Antibiotic prophylaxis is a highly effective way of managing rUTIs.

Three management regimens of antibiotic usage can be considered in women with rUTIs (Table 3)..$^{2,14}$

The choice of antibiotic should be based on confirmed urine culture and sensitivity results wherever possible, regional antibiotic resistance patterns as well as patient preferences and tolerance.

A Cochrane review of 10 studies of continuous low-dose antibiotic prophylaxis showed that the relative risk of clinical recurrence of UTI per patient year was 0.15 favouring antibiotics. ${ }^{15}$ Another way of stating this is that a woman with a history of rUTIs on antibiotic prophylaxis is 6.67 times less likely to have a UTI in a one-year period. The number needed to treat is 1.85 . The effect was not well maintained, with two studies showing that UTIs recurred and equalled the placebo arm after prophylaxis was ceased. ${ }^{15}$ Unfortunately, no clear evidence is available on the optimal duration of continuous prophylaxis, how often it should be repeated, the benefits post-prophylaxis, the threshold number of UTIs for starting prophylaxis or the optimal doses of different antibiotics.

In women with rUTIs associated with sexual intercourse, postcoital prophylaxis seems to be as effective as daily intake of antibiotics.
Patient-initiated antibiotics (with prior performance of an MSU) should be considered in a compliant patient with appropriate follow-up.

\section{Non-antibiotic treatment options for women with confirmed recurrent UTIs}

Vaginal oestrogen - The use of topical vaginal oestrogen in postmenopausal women helps reduce the rate of rUTIs. Vaginal oestrogen protects against colonisation by uropathogens via an increase in protective vaginal lactobacilli. ${ }^{16}$

Methenamine hippurate - This has a bacteriostatic effect in the urine. A Cochrane review in 2012 showed that short-term usage prevented rUTIs in women without urinary tract abnormalities or neuropathic bladder and was well tolerated with few adverse effects. ${ }^{17}$ The recommended dosage

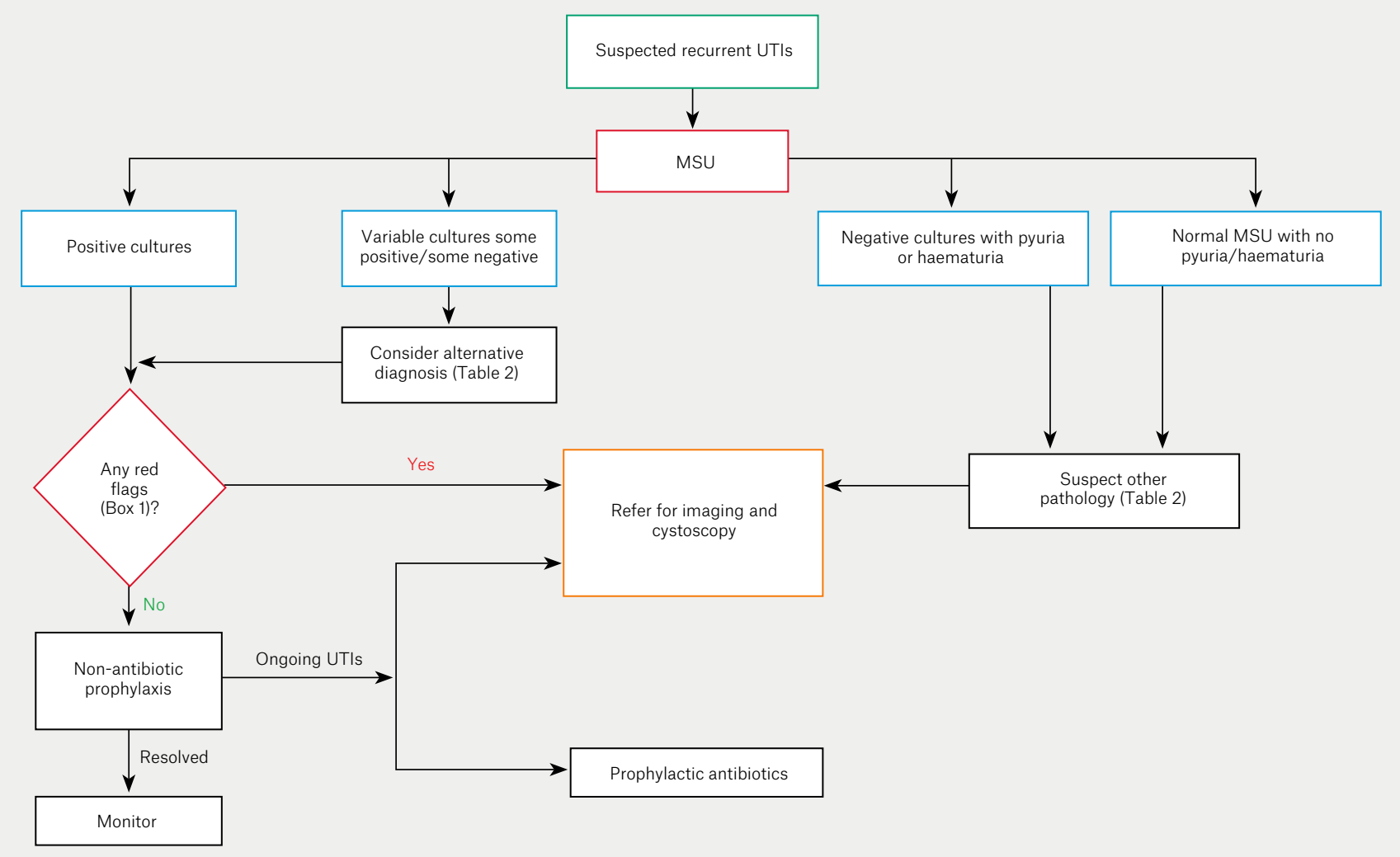

Figure 1. Management pathway in women with recurrent cystitis MSU, midstream urine; UTI, urinary tract infection 
of methenamine hippurate is $1 \mathrm{~g}$ twice daily, but studies have not defined optimal duration.

Cranberry products - The evidence for use of cranberry products is conflicting and may reflect the extreme variability in products and dosage and lack of standardised regimen. A Cochrane review in 2008 concluded that cranberry products could potentially decrease the frequency of symptomatic UTIs, but a re-analysis with further studies in 2012 did not show a significant benefit. ${ }^{18}$

D-mannose-D-mannose is thought to have an antibacterial activity by inhibiting the adherence of bacteria to urothelial cells and has been shown in limited randomised prophylaxis trials to reduce with the rate of UTI recurrence in women. Further studies are required before making definite recommendations. ${ }^{1}$

Probiotics (Lactobacillus spp.) A recent Cochrane review showed no convincing benefit of lactobacillus products in the prevention of rUTI. ${ }^{19}$ Further studies are needed because of the marked variability in preparations, which makes standardisation and comparison difficult.

Vaccines against urinary tract bacteriaThere are various oral, nasal and intravaginally administered vaccines that have been developed against $E$. coli. These have shown variable efficacy. OM-89 is an oral preparation of 18 different serotypes of heat-killed uropathogenic E. coli. It is the only vaccine recommended in guidelines ${ }^{1}$ as it has been shown to be more effective than placebo in reducing the rate of rUTIs in women in several randomised trials; however, its long-term efficacy is unclear.

\section{Women with symptoms of cystitis with variable urine cultures (both positive and negative urine cultures)}

Most of these women have true rUTIs and should be treated as for group 1 (women with confirmed rUTIs) but may require more extensive investigation to ensure that structural urinary tract problems (Table 1) are not present.

\section{Box 1. Red flags for early referral to a urologist for further assessment in women with recurrent urinary tract infections and cystitis symptoms}

- Urine cultures and symptoms do not match

- Persistent haematuria despite adequate control of infections

- Persistent sterile pyuria

- Ongoing pain

- Persistent bacteriuria despite appropriate antibiotic therapy

- Presence of urea-splitting bacteria (such as Proteus spp. and Pseudomonas spp.) on repeat cultures, which are associated with calculus disease

- A proven bladder or renal calculus on imaging

- Recurrent pyelonephritis

- Prior urinary tract surgery, incontinence surgery (eg sling procedure) or urinary tract trauma

- Prior abdominal or pelvic malignancy

- Prior pelvic radiation

- Neurological disease eg spinal cord injury, multiple sclerosis

- Obstructive voiding symptoms eg poor stream, hesitancy, incomplete emptying

- Poor bladder emptying on ultrasonography (especially residuals $>150 \mathrm{~mL}$ )

- Known renal tract abnormalities that may be contributing to recurrent infection eg vesicoureteric reflux, high-grade cystocele or prolapse, bladder outlet obstruction

- Immunocompromised patient

- Symptoms of a fistula between bladder and bowel eg pneumaturia (air in urine) or faecaluria (faeces in urine)

- Women who have not responded to preventive measures

- When the diagnosis of recurrent uncomplicated urinary tract infection is uncertain

Alternative differential diagnoses for rUTIs (Table 2 and Figure 1) should also be more strongly considered and investigated for with renal tract imaging and cystoscopy.

\section{Women with symptoms of cystitis and negative urine cultures with microhaematuria +/- micropyuria}

This group of women has definite bladder/ urethral irritation that is less likely to be due to standard bacterial infection if multiple urine cultures fail to show bacteria. They should not be treated with multiple courses of antibiotics unless other features on their assessment (eg cystoscopic findings) are suggestive of rUTI.

The alternative differential diagnoses for rUTIs (Table 2 and Figure 1) must be more strongly considered including urinary tract malignancy, calculus disease such as a vesicoureteric junction calculus and abnormalities of pelvic anatomy (eg high-grade pelvic organ prolapse, urethral diverticulum, other pelvic pathology).

As a result of the lack of diagnosis, these women require further investigations including upper tract imaging (renal tract ultrasonography $+/$ - computed tomography scan) and cystoscopy to exclude bladder and urethral pathology.

\section{Women with symptoms of cystitis with normal urine cultures (no pyuria or haematuria)}

In women who have completely normal urine tests with ongoing irritative voiding symptoms, alternative differential diagnoses apart from rUTI must be considered (Table 2 and Figure 1), especially urethral irritation after intercourse, sexually transmitted infections, overactive bladder syndrome, bladder hypersensitivity, interstitial cystitis/bladder pain syndrome, pelvic floor muscle dysfunction and abnormalities external to the urinary tract.

Further investigations should be considered to rule out bladder, urethral and functional abnormalities; these 
investigations include urinary tract imaging, cystoscopy and urodynamic studies depending on the woman's symptoms.

\section{Women with ongoing symptoms after a definite UTI (positive urine culture) who now have a negative urine culture}

After a prolonged or particularly symptomatically severe UTI, some women can have persistent urinary symptoms due to bladder hypersensitivity, which can be associated with pelvic floor muscle dysfunction. It is hypothesised that ongoing pain may be triggered by peripheral sensory and central nervous system sensitisation. ${ }^{20}$

When ongoing negative urine cultures are confirmed, treatment should focus on avoidance of further antibiotics, minimising intake of recognised bladder irritants such as caffeine, use of medications to reduce bladder hypersensitivity (eg low-dose amitriptyline), and pelvic floor physiotherapy to treat the abnormally high-tone pelvic floor muscles/pelvic floor muscle dysfunction.

Further investigations can be considered if a woman's symptoms persist despite these measures.

\section{Conclusion}

There are many potential causes of recurrent cystitis symptoms in women apart from rUTIs. MSU testing provides confirmation of bacterial infection in women to make the diagnosis of rUTIs. Both non-antibiotic and antibiotic-related measures can be used in the treatment of women with rUTIs. Other diagnoses must be considered in women with cystitis symptoms and sterile urine cultures, who also warrant further investigation.

\section{Key points}

- Repeated antibiotic treatment of presumed rUTIs without urine culture should be avoided.

- Repeat urine testing is important in establishing the diagnosis and good antibiotic stewardship.

- Early investigation of the urinary tract should be considered in women with complicated UTIs, women with uncomplicated UTIs who have atypical features or who are not responding to treatment, and women with red flag features.

- It is important to consider diagnoses other than rUTIs in women with symptoms and negative cultures.
- Women with rUTIs benefit from the use of non-antibiotic measures to prevent infection as well as the considered use of antibiotic prophylaxis.

\section{Authors}

Karen McKertich MBBS, FRACS (Urol), Urological Surgeon, Australian Urology Associates, Vic; Cabrini Health, Vic; The Alfred Hospital, Vic

Uri Hanegbi MBBS (Hons), FRACS (Urol), Urological Surgeon, Australian Urology Associates, Vic; Cabrini Health, Vic; The Alfred Hospital, Vic

Competing interests: None.

Funding: None.

Provenance and peer review: Commissioned, externally peer reviewed.

Correspondence to:

reception@aua.com.au

\section{References}

1. Bonkat $G$, Bartoletti $R$, Bruyère $F$, et al. EAU Guidelines on urological infections. Arnhem, NL: EAU Guidelines Office, 2020.

2. Expert Group for Antibiotic. Antibiotic choice for urinary tract infection in adults. In: eTG complete [Internet]. West Melbourne, Vic: Therapeutic Guidelines Limited, 2020.

3. Kass EH. Bacteriuria and pyelonephritis of pregnancy. Arch Intern Med 1960:105:194-98. doi: 10.10001/archinte.1960.00270140016003.

4. Hooton TM, Roberts PL, Cox ME, Stapleton AE. Voided midstream urine culture and acute cystitis in premenopausal women. $N$ Engl J Med 2013;369(20):1883-91. doi: 10.1056/ NEJMoa1302186

5. Bent S, Nallamothu BK, Simel DL, Fihn SD, Saint S. Does this woman have an acute uncomplicated urinary tract infection?

\section{Table 3. Antibiotic regimens used for recurrent urinary tract infections ${ }^{2,14}$}

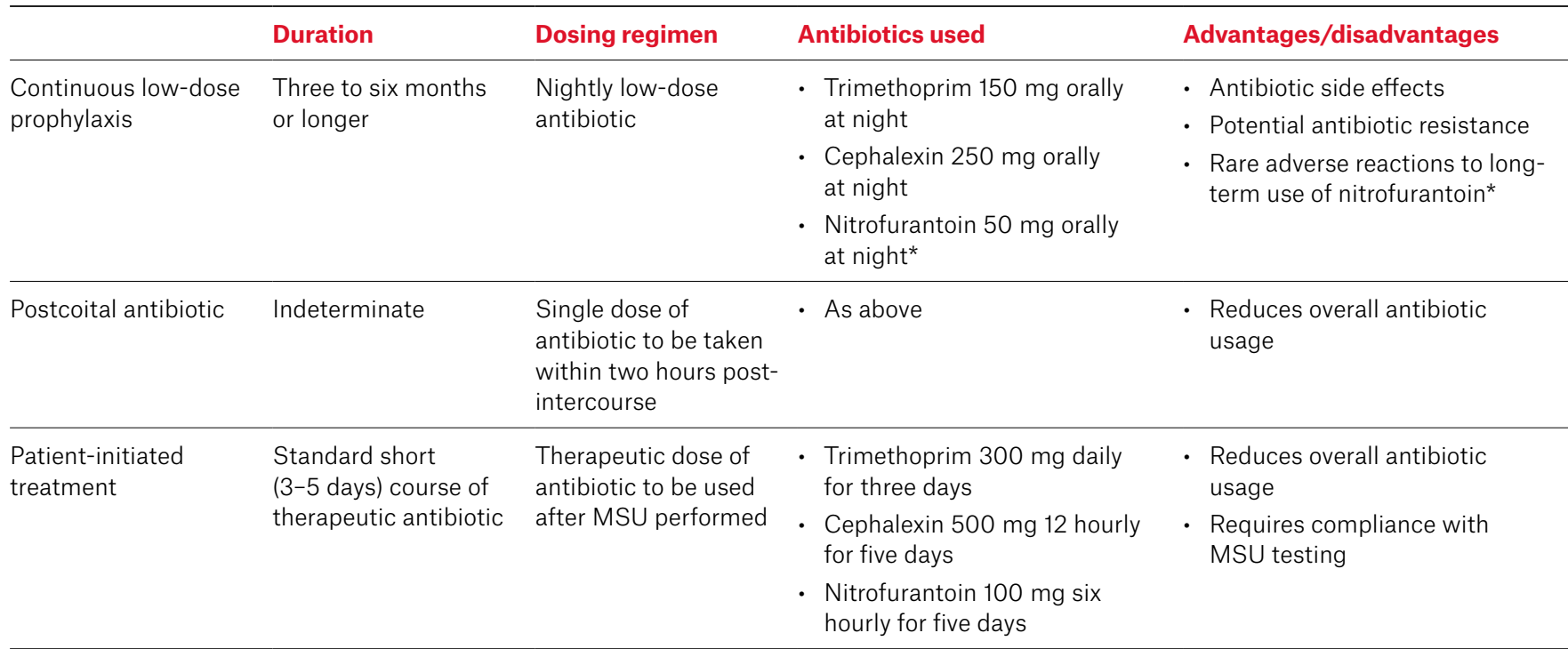

${ }^{*}$ Care must be taken with long-term nitrofurantoin usage because of rare adverse effects such as pulmonary toxicity, hepatotoxicity and peripheral neuropathy ${ }^{2}$ MSU, midstream urine 
JAMA 2002;287(20):2701-10. doi: 10.1001/ jama.287.20.2701.

6. Anger J, Lee U, Ackerman AL et al. Recurrent uncomplicated urinary tract infections in women: AUA/CUA/SUFU guideline. J Urol 2019;202(2):282-89. doi: 1097/ JU.0000000000000296.

7. Dason S, Dason JT, Kappor A. Guidelines for the diagnosis and management of recurrent urinary tract infection in women. Can Urol Assoc J 2011;5(5):316-22. doi: 10.5489/cuaj.11214.

8. Nygaard I, Kinder M. Thirst at work - An occupational hazard? Int Urogynaecol J Pelvic Floor Dysfunct 1997;8(6):340-43. doi: 10.1007/ BF02765593.

9. Kunin CM, Polyak F, Postel E. Periurethral bacterial flora in women. Prolonged intermittent colonization with Escherichia coli. JAMA 1980;243(2):134-39.

10. Nicolle LE, Harding GK, Preiksaitis J, Ronald AR. The association of urinary tract infection with sexual intercourse. J Infect Dis 1982;146(5):579-83. doi: 10.1093/infdis/146.5.579.

11. Hooton TM, Scholes D, Hughes JP, et al. A prospective study of risk factors for symptomatic urinary tract infection in young women. N Engl J Med 1996:335(7):468-74. doi: 10.1056/NEJM199608153350703.

12. Scholes D, Hooton TM, Roberts PL, Stapleton AE, Gupta K, Stamm WE. Risk factors for recurrent urinary tract infection in women. J Infect Dis 2000;82(4):1177-82. doi: 10.1086/315827.

13. Hooton TM, Stapleton AE, Roberts PL, et al. Perineal anatomy and urine-voiding characteristics of young women with and without recurrent urinary tract infections. Clin Infec Dis 1999;29(6):1600-01. doi: 10.1086/313528.

14. Australian Medicines Handbook. Anti-infectivesdrug choice for selected infections. Adelaide, SA: Australian Medicines Handbook, 2020. Available at https://amhonline.amh.net.au [Accessed 25 February 2020].

15. Albert X, Huertas I, Pereiró II, Sanfélix J, Gosalbes V, Perrota C. Antibiotics for preventing recurrent urinary tract infection in nonpregnant women. Cochrane Database Syst Rev 2004;(3):CD001209. doi: 10.1002/14651858. CD001209.pub2.

16. Perotta C, Aznar M, Mejia R, Albert X, Ng CW. Oestrogens for preventing recurrent urinary tract infection in postmenopausal women. Cochrane Database Syst Rev 2008;(2):CD005131. doi: 10.1002/14651858.CD005131.pub2.

17. Lee BS, Bhuta T, Simpson JM, Craig JC. Methenamine hippurate for preventing urinary tract infections. Cochrane Database Syst Rev 2012;10(10):CD003265. doi: 10.1002/14651858. CD003265.pub3.

18. Jepson RG, Williams G, Craig JC. Cranberries for preventing urinary tract infections. Cochrane Database Syst Rev 2012;10(10):CD00132. doi: 10.1002/14651858.CD001321.pub5.

19. Schwenger EM, Tejani AM, Loewen PS. Probiotics for preventing urinary tract infections in adults and children. Cochrane Database Syst Rev 2015;(12):CD008772. doi: 10.1002/14651858. CD008772.pub2.

20. Rosen JM, Klumpp DJ. Mechanisms of pain from urinary tract infection. Int J Urol 2014;21 Suppl 1(0 1):26-32. doi: 10.1111/iju.12309. 